\title{
On the predictability limit of convection models of the Earth's mantle
}

\author{
L. Bello ${ }^{a, *}$, N. Coltice ${ }^{a, b}$, T. Rolf ${ }^{1}$, P.J. Tackley ${ }^{1}$ \\ ${ }^{a}$ Laboratoire de Géologie de Lyon, Université Claude Bernard Lyon 1, \\ Ecole Normale Supṕrieure de Lyon, France. \\ ${ }^{b}$ Institut Universitaire de France, France. \\ ${ }^{c}$ EarthByte Group, University of Sydney, Sydney, Australia
}

Cite as Bello, L., Coltice, N., Rolf, T., Tackley, P. J. (2014). On the predictability limit of convection models of the Earth's mantle. Geochemistry, Geophysics, Geosystems, 15(6), 2319-2328.

\begin{abstract}
Reconstructing convective flow in the Earth's mantle is a crucial issue for a diversity of disciplines, from seismology to sedimentology. The common and fundamental limitation of these reconstructions based on geodynamic modelling is the unknown initial conditions. Because of the chaotic nature of convection in the Earth's mantle, errors in initial conditions grow exponentially with time and limit forecasting and hindcasting abilities. In this work we estimate for the first time the limit of predictability of Earth's mantle convection. Following the twin experiment method, we compute the Lyapunov time (i.e. e-folding time) for state-of-the art 3D spherical convection models, varying rheology and Rayleigh number. Our most Earth-like and optimistic solution gives a Lyapunov time of $136 \pm 13$ My. Rough estimates of the uncertainties in best guessed initial conditions are around $5 \%$, leading to a limit of predictability for mantle convection of $95 \mathrm{My}$. Our results suggest that error growth could produce unrealistic convective structures over timescales shorter than that of Pangea dispersal.
\end{abstract}

\section{Introduction}

Reconstructing the history of convection in the Earth's mantle is a fundamental issue for a diversity of disciplines. The evolving density structure within the planet controls, for instance, the evolution of sea-level, vertical motion of continents or Earth's moment of inertia. In the past 15 years, the primary strategy for establishing the history of mantle flow has been to force convection and temperature redistribution by imposing surface velocities derived from plate tectonic reconstructions [e.g. ?]. Time-dependent surface kinematics drives

\footnotetext{
*Corresponding author: Lea Bello

Email address: Lea.bello@ens-lyon.fr (L. Bello)
}

the large-scale transport of thermal and chemical heterogeneities. This approach was successful in predicting, to first order, seismic velocity variations [?], deep mantle chemical heterogeneities [?] and dynamic topography [?]. Despite difficulties in reconstructing plate kinematics before $200 \mathrm{Ma}$ [?], ? attempted to push mantle convection reconstructions back to $450 \mathrm{Ma}$.

The common and crucial limitation of these models is the unknown initial conditions. In the absence of constraints, the initial condition for the thermal field is commonly approximated by a statistically steady state solution, obtained by imposing the most ancient known surface velocity field at all times [see ?]. To overcome this problem, backward advection of a seismically derived temperature and density field has been 
used, but was limited to several tens of million years, over which thermal diffusion is considered negligible [???]. To take into account the effects of thermal diffusion, variational data assimilation methods have been pioneered to compute the initial condition up to $100 \mathrm{My}$ ago [???]. These methods employ a present-day thermal field derived from seismic tomography for the assimilated data, and reconstructed surface velocities to drive the convective flow.

Convection in the Earth's mantle is chaotic [??]. As a consequence, two initially very close convective states diverge quickly from each other in time to ultimately produce two uncorrelated thermal structures [?]. This is known as the Butterfly effect: a small wobbling of the Iceland plume could set off subduction in the Pacific. Hence, there is an intrinsic limit of predictability for any chaotic system, which any mantle convection reconstruction strategy faces. Backward advection is limited not only by thermal diffusion, but also by the chaotic nature of the flow. So is data assimilation, and even more reconstructions, starting from less constrained solutions.

The predictability limit of mantle convection has never been quantified. In this paper, we follow the twin experiment approach initially developed by ? and used in climate sciences [?], geomagnetism [?] and solar dynamics [?], to evaluate the range of predictability of 3D spherical convection models with diverse rheologies.

\section{Limit of predictability}

The time-dependence of convection at high Rayleigh number is strong enough to develop a chaotic regime. The presence of lateral viscosity variations in the Earth's mantle results in a significant toroidal component of the surface velocity field [??] that further enhances the chaotic nature of the system [?]. Deterministic chaos of mantle convection implies that two slightly different initial states, evolving according to the same physical laws and same material properties, will result in two significantly different states after some time. Predictions based on mantle convection calculations are therefore intrinsically limited to a certain duration. The limit of predictability depends on the uncertain- ties in initial conditions, the growth rate of the errors in the convection calculations, and the tolerance error of the predicted state.

? described experimentally the growth rate of errors in atmospheric models. Later, using the theory of dynamical systems, physicists proved that the differential equations governing the error growth in convective atmospheric systems can be linearized under certain conditions [e.g. ??]. Hence, the evolution with time $t$ of a temperature perturbation $E(t)$, initially equal to $E(0)$ and sufficiently small, can be approximated by:

$$
E(t)=E(0) e^{\lambda t}\left[1+\mathcal{O}\left(e^{-\left(\lambda-\lambda_{2}\right) t}\right)\right] .
$$

Here $\lambda$ and $\lambda_{2}$ are the two largest characteristic Lyapunov exponents of the system [?]. For chaotic convection, $\lambda$ is positive, i.e. the error grows exponentially as $\exp (\lambda \mathrm{t})$. We can thus define a characteristic time of the system, the Lyapunov time: $\tau=1 / \lambda$. The limit of predictability $t_{\text {pred }}$ is linearly proportional to $\tau$ and depends on the initial and tolerance errors on the prediction, $E(0)$ and $\Delta$ respectively [e.g. ?]:

$$
t_{\text {pred }}=\tau \ln \frac{\Delta}{E(0)}
$$

In order to evaluate $t_{\text {pred }}$ for mantle convection, we first need to evaluate the Lyapunov time. The classic methodology to do so is the twin experiment method, developed by ? for dynamic meteorology and repeatedly used in various fields of geophysics, from climate sciences [?] to geomagnetism [?] and solar dynamics [?]. A twin experiment is defined as the comparison of two initially very close dynamical trajectories. The timescale of divergence of these two trajectories provides the Lyapunov time by integration of Eq. (??).

First, we use a convection model to generate a statistically steady-state solution for the temperature field. This state is used as the initial condition for one of the twins, referred hereafter as the reference twin. We create the initial condition of the other twin by adding a perturbation to the initial condition of the reference twin. Here, we introduce random perturbations of temperature uniformally distributed in space, which produce a white noise, i.e. errors at all scales from the smallest to the largest. The Lyapunov exponent 
is intrisic and expected not to depend on the lengthscale of the error, as verified by ? for the Earth's core.

The magnitude of the local perturbations is required to be small for Eq. (??) to be valid. We set the magnitude to $0.01-1 \%$ of the average temperature depending on our calculation, making sure negative temperatures are filtered. The corresponding volume averaged differences of the temperature fields of the two twins are $0.002-0.2 \%$ (see Table 1 ). We monitor the value of this difference through time:

$$
E(t)=\int_{V_{\Omega}} \frac{\left|T_{p}(\mathbf{x}, t)-T(\mathbf{x}, t)\right|}{T(\mathbf{x}, t)} \frac{\mathrm{d} V(\mathbf{x})}{V_{\Omega}}(3)
$$

where $T(\mathbf{x}, t)$ and $T_{p}(\mathbf{x}, t)$ represent the temperature at position $\mathrm{x}$ of the reference twin and the perturbed twin, respectively. $V_{\Omega}$ is the volume of the model in which the temperature is not imposed by boundary conditions. We then use a least-squares method to fit the evolution of $E(t)$ using Eq. (??). The end of the exponential growth can be difficult to estimate precisely. Hence, we fit the Lyapunov time for all time windows in the exponential growth phase having a number of points $>10$ and a low misfit, and we choose the mode of the fitted Lyapunov times ( $>1000)$ to define the Lyapunov time of the twin experiment.

For each set of convection parameters, we proceed to multiple evaluation of the Lyapunov time by computing a family of twins differing in the magnitude of the initial perturbation. In general, we compute 3 twin experiments for a given set of convection parameters. We define the Lyapunov time for this set of convection parameters as the average of the Lyapunov times of this family of twin experiments. The computational cost of 3D spherical convection models was a limiting factor for the number of numerical solutions we computed. Varying the initial conditions for the reference twin, the type of perturbation, and computing more twin experiments would improve the accuracy of our estimates.

Once the Lyapunov time is estimated for a variety of dynamic models, we evaluate $t_{\text {pred }}$ for mantle convection by estimating uncertainties in the initial conditions and the tolerance error relevant to the Earth.

\section{Convection Model}

We compute time-dependent solutions for incompressible mantle convection in 3D spherical geometry using the code StagYY [?]. The resolutions used here are $45 \mathrm{~km}$ close to the surface for cases with Rayleigh numbers lower than or equal to $10^{6}$, and $23 \mathrm{~km}$ for higher Rayleigh numbers. Here, the Rayleigh number Ra is given by:

$$
\mathrm{Ra}=\frac{\rho g \alpha \Delta T L^{3}}{\kappa \eta_{0}},
$$

where $\rho, g, \alpha, \Delta T, L, \kappa$ and $\eta_{0}$ are density, gravitational acceleration, thermal expansivity, temperature scale, mantle thickness, thermal diffusivity and reference viscosity obtained at non-dimensional temperature $T=1$, respectively. Because computational power is limited, we have restricted our study to purely internally heated convection, neglecting the effects of hot plumes. The non-dimensional internal heating rate $H$ is chosen for each calculation to obtain a non-dimensional temperature drop of 1 across the mantle (see Table ??). We have further focused the exploration of the parameter space on rheological aspects, since choosing a rheology for mantle rocks has a decisive impact on the time-dependent structure of convective flow and the strength of the toroidal component of the surfave velocity field. In this study, we present 32 different 3D spherical calculations we computed, resulting in 22 twin experiments (see Table ??).

The models with uniform viscosity (named ISO for isoviscous) displays short-lived cold plumes operating at small scales (Fig. ??a). We compute models with viscosity increasing in the lower mantle (named LV for layered viscosity), as required by geophysical constraints [??]. Because the radial viscosity structure remains difficult to resolve finely, we implement a gradual viscosity increase by a factor of 40 between 800 and $1000 \mathrm{~km}$ depth. In these calculations, strongly time-dependent, drip-like small-scale instabilities persist in the upper mantle, but the more sluggish lower mantle introduces a longwavelength flow component [??], that ultimately dominates the power spectrum of the temperature field (see Fig. ??b). 
Lateral viscosity variations are required to explain surface features on Earth like the toroidal component of the surface velocity field [??]. Hence, we investigated models with pseudoplasticity, in which the viscosity is temperatureand stress-dependent (named PL for plate-like behavior). When the stress exceeds the yield stress, the viscosity is decreased to reduce the stress back to the yield stress. We used the same formulation as in ?, and indeed observed that convection with plate-like behavior leads to stable large-scale flow (Fig. ??c) with a toroidal component of the surface velocity amounting to $23 \%$ in this case. Length scales of the flow are here even larger than those produced by layered viscosity alone, as described by ?.

We also computed solutions with both pseudo-plasticity and a viscosity dependence with depth (named PLLV for plate-like behavior and layered viscosity), the viscosity for a given temperature increasing by a factor of 30 between 800 and $1000 \mathrm{~km}$ depth. The overall structure of the flow is similar to cases with plate-like behavior, but the downwellings are slowed down and buckle in the more sluggish deeper mantle. The toroidal component of the surface velocity is close to $38 \%$.

We finally computed solutions with pseudoplasticity and continental rafts (named PLC for plate-like behavior and continents), following ? but with Earth-like shapes and starting from a configuration similar to Pangea, $200 \mathrm{My}$ ago. The combination of plate-like behavior and continental rafts produces mantle convection that matches, to first order, basic tectonic features observed on Earth [??]. Large scale convection is also developed in these simulations (Fig. ??d) with a toroidal component of $41 \%$ of the surface velocity field.

To compare our calculations both to the Earth and to each other, time is scaled by the transit time $t_{t}=L / v_{\text {surf }}$ where $v_{\text {surf }}$ is the time and space averaged surface velocity. Assuming a surface velocity of $3.4 \mathrm{~cm} \cdot \mathrm{y}^{-1}$ and a mantle thickness of $2900 \mathrm{~km}$, the transit time is $85 \mathrm{My}$ for the Earth's mantle. Thus, time is dimensionalized as $t=t^{\text {Model }} \times t_{t}^{\text {Earth }} / t_{t}^{\text {Model }}$.

\section{Sensitivity of the Lyapunov Time}

The initial error grows in three main phases: (1) a short diffusion phase where error decreases, (2) an exponential growth phase and (3) a saturation phase (Fig. ??). During the first phase, the smallest scale perturbations are smeared out by thermal diffusion before advection becomes significant. The duration of this phase does not depend on the amplitude of the initial perturbation.

The growth phase consists of the transport and dissemination of the error described by Eq. (??), causing significant and global modifications in the flow structure. The duration of this phase, much longer than the diffusion phase, depends on the amplitude of the initial error: the smaller $E(0)$, the longer the exponential growth. However, the Lyapunov time does not depend on $E(0)$ (as long as it is small, see Fig. ??) as predicted by Eq. (??).

The saturation phase starts when the error does not grow anymore. From then on, the perturbed solution evolves with no apparent correlation to the reference solution and initial twins cannot be recognized. The value of the saturation does not depend on $E(0)$ and reaches $\sim 10 \%$ in our models. Scaled to the Earth's mantle, this value represents an average local temperature difference of $\sim 150 \mathrm{~K}$ between perturbed and reference solutions.

We obtain the Lyapunov time through fitting the slope of the exponential growth stage. The larger the initial error, the faster the saturation is reached, and the less precise the determination of the Lyapunov time. Averaging individual Lyapunov times obtained for the initial errors $E(0)=$ $0.2 \%, 0.02 \%$, and $0.002 \%$, the Lyapunov time is $35 \pm 0.5 \mathrm{My}$ and $236 \pm 36 \mathrm{My}$ for ISO and $\mathrm{PL}$ models respectively (Table ??).

Fig. ?? shows the influence of the Ra on the Lyapunov time for isoviscous convection and for convection with plate-like behavior. We performed twin experiments for $\mathrm{Ra}=10^{5}, 3 \times 10^{5}$, $10^{6}, 5 \times 10^{6}$ and $10^{7}$ for a diversity of initial perturbations (Table ??). As seen in Fig. ??, where experiments conducted with $E(0)=$ $0.2 \%$ are presented, the rate of exponential growth increases with $\mathrm{Ra}$ and tends to saturate at high $\mathrm{Ra}$. In isoviscous models, $\tau$ drops 
from $78 \mathrm{My}$ at $\mathrm{Ra}=3 \times 10^{5}$ (ISO1) to $35 \mathrm{My}$ at $\mathrm{Ra}=10^{7} \quad(\mathrm{ISO} 4,5,6)$, while in models with plate-like behavior, $\tau$ drops from $\sim 400 \mathrm{My}$ at $\mathrm{Ra}=10^{5}(\mathrm{PL} 1,2)$ to $\sim 220 \mathrm{My}$ at $\mathrm{Ra} \geq 3 \times 10^{5}$ (PL3,4,5,6,7,8).

The decrease of the Lyapunov time to a minimum value corresponds to the evolution towards fully developed chaotic regime. For Ra higher than $5 \times 10^{6}$ in ISO cases, the mantle flow structure shows transient formation and development of new boundary-layer instabilities. This regime of thermal turbulence [?], also called "plume-dominated" regime [?], is strongly timedependent and rapidly fluctuating, which ensures deterministic chaos. In this convection regime, the non-dimensional Lyapunov time depends only on the inverse of convective velocities $\left(v \alpha \mathrm{Ra}^{2 / 3}\right)$ and reaches its minimum value. Hence, choosing to scale with the transit time implies that the dimensional Lyapunov time is not dependent on $\mathrm{Ra}$ in the fully chaotic regime. The scaling of mixing times for mantle convection is similar [?].

Hence, we measured $\tau$ when $\mathrm{Ra}$ is large enough to ensure a fully chaotic regime, for each rheology considered here. The corresponding Ra is below Earth's values, so we were able to reach an Earth-like chaotic regime despite our calculation limitations. The transition into fully developed chaos occurs at a lower Ra for PL than for ISO models (Fig. ??). The existence of a significant toroidal component of the surface velocity field in models with plate-like behavior is probably fundamental in this phenomenon. We obtained $\tau=210 \pm 26 \mathrm{My}$ and $\tau=236 \pm 36 \mathrm{My}$ for $\mathrm{PL}$ calculations at $\mathrm{Ra}=3 \times 10^{5}$ and $\mathrm{Ra}=10^{6}$, respectively. Because the convective structures (temperature anomalies with respect to the adiabatic state) are thinner and thermal mixing is more efficient at higher $\mathrm{Ra}$, the saturation error slightly decreases with convective vigor (Fig. ??).

The Lyapunov time strongly depends on mantle rheology (Fig. ??). Each twin experiment is carried out at Ra high enough to ensure that $\tau$ has reached its minimum value $\left(\mathrm{Ra}=10^{7}\right.$ for ISO and LV models, $\mathrm{Ra}=10^{6}$ for PL, PLLV and PLC models). In the four cases presented in Fig. ??, $\mathrm{E}(0)$ is $0.2 \%$, but the corresponding Lyapunov times were also reproduced by twin experiments with initial perturbations of $0.02 \%$ and $0.002 \%$ (Table ??). ISO has the shortest $\tau(35 \pm 0.5 \mathrm{My})$ whereas the PL displays the longest $\tau$ (236 $\pm 36 \mathrm{My}$ ).

The LV case introduces an additional degree of complexity in estimating $\tau$ as there are two Lyapunov times involved, namely $57 \pm 4 \mathrm{My}$ and $118 \pm 4$ My (Fig. ??). The shorter dominates at infinitesimal errors, and the longer dominates at larger errors. This phenomenon is typical for a chaotic system involving two time scales: the shorter $\tau$ is associated with small-scale dynamics and saturates quickly, then the longer $\tau$, associated with the large-scale flow, takes over [?]. In the LV cases, small-scale and strongly time-dependent instabilities in the upper mantle (Fig. ??) enforce quick error growth. Such structures do not develop in the more sluggish lower mantle. The shorter $\tau$ is close to that of the ISO case as expected, and the longer $\tau$ corresponds to the large-scale component, somewhat similar to the PL case, which is large-scale as well.

When plate-like behavior and layered viscosity are combined, the error grows with the same type of evolution as in the PL cases. In contrast to the LV cases, there are no small-scale instabilities in the upper mantle and only one Lyapunov time is required: $\tau=119 \pm 6 \mathrm{My}$, which is even shorter than that of PL. The fact that this Lyapunov time is almost the same as the longer $\tau$ of LV is probably coincidental, since the structure and time-dependence of these two flows are extremely different. When continents are added to the $\mathrm{PL}$ model, $\tau$ is $136 \pm 13 \mathrm{My}$. The Lyapunov times for PLLV and PLC are both shorter than that of PL probably because they both have a significantly higher toroidal component of the surface velocity field than PL (38\% and $41 \%$ versus $23 \%$, respectively), which enhances chaoticity.

\section{Limit of predictability for the Earth's Mantle}

In addition to the Lyapunov time, computing the limit of predictability requires knowledge of the initial error $E(0)$ and of the tolerance error $\Delta$ for the prediction (Eq.(??)). $\Delta$ is taken here to be the saturation of the error observed in our twin experiments. It weakly depends on rheology, slightly decreases with $\mathrm{Ra}$ and remains 
close to $10 \%$.

The most Earth-like model would integrate pseudo-plasticity, layered viscosity, continents and heating from the core, as well as additional compexities of mantle convection (e.g. grainsize rheology, compressibility, phase changes, chemical heterogeneity). But this is a first attempt to estimate the predictability limit of convection in the Earth's mantle and computing such model remains beyond the scope of this study. We therefore try to estimate a Lyapunov time for the Earth's mantle using the calculations presented here.

We have shown that the PLC and PLLV models have similar Lyapunov times, both displaying long wavelength flow and a high toroidal component of their respective surface velocity fields. We assume here that introducing continents in the PLLV model would not produce a longer Lyapunov time. We also do not expect a moderate amount of basal heating added to the PLC or PLLV models to alter the Lyapunov time. Indeed, the study of chaotic mixing shows that convection heated from the base displays Lagrangian Lyapunov exponents similar to those with internal heating only, as long as the fully chaotic regime is reached [?]. Hence, we assume here that our best and most optimistic Lyapunov time for Earth's mantle convection is that of PLC: $136 \pm 13 \mathrm{My}$.

Given this value, a precision of $10^{-16}$ on the initial temperature field would be necessary to predict 4 Gy of Earth's evolution, which makes this exercise impossible. We focus here on predictions published in the last 15 years that span over 30-75 My for backward advection [???], 75-100 My for data assimilation [???], and 250-450 My for forward convection calculations [???]. To remain below the tolerance error throughout the whole integration time, the error on the initial conditions has to be $<8 \%$ for $30 \mathrm{My},<5 \%$ for $100 \mathrm{My}$ and $<0.4 \%$ for $450 \mathrm{My}$.

The best guesses for initial conditions used for forecasts and hindcasts, up to this day, are 3D temperature fields derived from tomographic models. The errors on such temperature fields come both from uncertainties in the tomographic models and from the conversion of seismic velocities into temperature. ? compared 3 tomographic models for $\mathrm{P}$-waves and 7 models for S-waves, showing correlations of 0.5 to 0.9 between different models depending on depth. Local differences between models of S-velocity anomalies are consistently over $0.5 \%$, which can be considered here as a strict minimum. For a known mineralogical model, such deviation corresponds to an uncertainty of $100 \mathrm{~K}$ on the local temperature above $400 \mathrm{~km}$, and $250 \mathrm{~K}$ in the shallow lower mantle [?]. Additional uncertainties have to be taken into account when converting the seismic velocities into temperature: composition is not well-known and phase diagrams are not determined with absolute precision [??]. Such considerations suggest that the errors in a starting temperature field derived from tomographic models is already as large as the tolerance error of the convection model. However, tomographic models also agree on coherent structures at the larger scales, consistent with sinking slabs [?] for instance. Hence, we propose that the average local uncertainty on the temperature could be lower and around 5\%. A careful analysis of the uncertainties is required to reach a more accurate estimate.

Using $E(0)=5 \%$ leads to a limit of predictability $t_{\text {pred }}=95 \mathrm{My}$. As a consequence, error growth operates on the timescale shorter than that of Pangea dispersal. Proposing a scenario for a future supercontinent therefore seems out of reach yet. We also show here that backward reconstruction of a thermal field is limited not only by thermal diffusion [???]. It is evenly limited by backward advection of initial uncertainties that grow exponentially to reach the saturation error within a timescale comparable to that of these calculations. However, with this study we cannot predict how different wavelengths are affected.

For models with imposed surface velocities [??, among others], it is possible that driving the flow could help to impede the divergence from the solution relevant to the Earth, despite the significant time-dependence and toroidal component of imposed surface velocities. De facto, some of these models were successful for predictions of seismic velocity variations [?], deep mantle chemical heterogeneities [?] and dynamic topography [?]. Despite these important successes, uncertainties in the surface velocities for deep time reconstructions introduce new sources 
of error in the system, which will ultimately grow as well. Data assimilation strategies that use a tomographic thermal field as input for defining present day structure [??] should also be limited in time by the growth of uncertainties in a chaotically convecting mantle.

\section{Conclusions}

Predictions of the past or future convective structure of the Earth's mantle are intrinsically limited in time because of the chaotic nature of mantle convection. We have used the twin experiment method to evaluate the Lyapunov time, which corresponds to the characteristic time of exponential growth of the error. This time is proportional to the inverse of the velocity when the convective regime is fully chaotic. It depends on the rheology and is maximum with plate-like behavior, because of the existence of long-living stable structures. The presence of a high toroidal component in the surface velocity field reduces the Lyapunov time. Our most optimistic estimate for the Earth's mantle is $136 \pm 13 \mathrm{My}$, but our models would require improvements to obtain a more accurate value (basal heating and compressibility, among other features).

The limit of predictability of the Earth's mantle increases with the Lyapunov time, but decreases with uncertainties in initial conditions. For most models used for convection reconstructions, the best guesses for initial conditions in forward or backward integration are derived from tomographic models. The uncertainties in these initial conditions are difficult to estimate, especially as a function of wavelength. A rough estimate would suggest a limit of predictability of $95 \mathrm{My}$. Hence, because of the chaotic nature of the flow, the error growth is almost as limiting as thermal diffusion for backward advection. Our results suggest that uncertainties in initial conditions could produce unrealistic structures in convection reconstructions over times comparable to Pangea dispersal. We propose that future convection reconstructions are published together with a computation of their limit of predictability (i.e. error analysis). This type of information will be extremely useful to estimate their level of confidence.

\section{Acknowledgements}

We are thankful to the editor and both reviewers for their comments and questions, which greatly contributed to the improvement of the manuscript. Fruitful discussions were had with A. Fournier, G. Hulot, Y. Ricard, M. Ulvrova and N. Flament. The research leading to these results has received funding from Institut Universitaire de France, ENS de Lyon, and the European Research Council within the framework of the SP2-Ideas Program ERC-2013-CoG, under ERC Grant agreement number 617588. Calculations were performed on LGLTPE Seisglob high-performance computing cluster.

\section{References}

Arnold, L. (1998), Measurable Dynamical Systems, in Random Dynamical Systems, Springer Monographs in Mathematics, chap. IV Apendix, pp. 535-550, Springer Berlin Heidelberg, doi:10.1007/ 978-3-662-12878-7.

Becker, T. W., and L. Boschi (2002), A comparison of tomographic and geodynamic mantle models, Geochemistry, Geophysics, Geosystems, 3(2001GC000168), doi:10.1029/2001GC000168.

Boffetta, G. (2002), Predictability: a way to characterize complexity, Physics Reports, 356(6), 367-474, doi: 10.1016/S0370-1573(01)00025-4.

Boffetta, G., P. Giuliani, G. Paladin, and A. Vulpiani (1998), An Extension of the Lyapunov Analysis for the Predictability Problem, Journal of the Atmospheric Sciences, 55(23), 3409-3416, doi:10.1175/ 1520-0469(1998)055\$ \$3409:AEOTLA $\$\rangle$ \$2.0.CO;2.

Bunge, H., M. Richards, C. Lithgow-Bertelloni, J. Baumgardner, S. Grand, and B. Romanowicz (1998), Time scales and heterogeneous structure in geodynamic earth models, Science, 280(5360), 91-5.

Bunge, H.-p., M. A. Richards, and J. R. Baumgardner (1997), A sensitivity study of three-dimensional spherical mantle convection at $10^{8}$ Rayleigh number: Effects of depth-dependent viscosity, heating mode, and an endothermic phase change, Journal of Geophysical Research, 102(B6), 11,991, doi:10.1029/96JB03806.

Bunge, H.-P., C. R. Hagelberg, and B. J. Travis (2003), Mantle circulation models with variational data assimilation: inferring past mantle flow and structure from plate motion histories and seismic tomography, Geophysical Journal International, 152(2), 280-301, doi: 10.1046/j.1365-246X.2003.01823.x.

Cammarano, F., S. Goes, P. Vacher, and D. Giardini (2003), Inferring upper-mantle temperatures from seismic velocities, Physics of the Earth and Planetary Interiors, 138(3-4), 197-222, doi:10.1016/ S0031-9201(03)00156-0.

Coltice, N., and J. Schmalzl (2006), Mixing times in the mantle of the early Earth derived from 2-D and 
3-D numerical simulations of convection, Geophysical Research Letters, 33(23), L23,304, doi:10.1029/ 2006GL027707.

Coltice, N., T. Rolf, P. J. Tackley, and S. Labrosse (2012), Dynamic causes of the relation between area and age of the ocean floor., Science, 336(6079), 3358, doi:10.1126/science.1219120.

Coltice, N., M. Seton, T. Rolf, R. Müller, and P. Tackley (2013), Convergence of tectonic reconstructions and mantle convection models for significant fluctuations in seafloor spreading, Earth and Planetary Science Letters, 383, 92-100, doi:10.1016/j.epsl.2013.09.032.

Conrad, C. P., and M. Gurnis (2003), Seismic tomography, surface uplift, and the breakup of Gondwanaland: Integrating mantle convection backwards in time, Geochemistry, Geophysics, Geosystems, 4(3), 1031, doi: 10.1029/2001GC000299.

Davies, D. R., S. Goes, J. Davies, B. Schuberth, H.P. Bunge, and J. Ritsema (2012), Reconciling dynamic and seismic models of Earth's lower mantle: The dominant role of thermal heterogeneity, Earth and Planetary Science Letters, 353-354, 253-269, doi: 10.1016/j.epsl.2012.08.016.

Ferrachat, S., and Y. Ricard (1998), Regular vs. chaotic mantle mixing, Earth and Planetary Science Letters, 155(1-2), 75-86, doi:10.1016/S0012-821X(97) 00200-8.

Flament, N., M. Gurnis, and R. D. Muller (2013), A review of observations and models of dynamic topography, Lithosphere, 5(2), 189-210, doi:10.1130/L245.1.

Goswami, B. N., and J. Shukla (1991), Predictability of a Coupled Ocean-Atmosphere Model, Journal of Climate, 4(1), 3-22, doi:10.1175/1520-0442(1991) 004\$<\$0003:POACOA $\$\rangle 2.0 . \mathrm{CO} ; 2$.

Hager, B. H., and R. J. O'Connell (1979), Kinematic models of large-scale flow in the Earth's mantle, Journal of Geophysical Research, 84(B3), 1031, doi: 10.1029/JB084iB03p01031.

Hulot, G., F. Lhuillier, and J. Aubert (2010), Earth's dynamo limit of predictability, Geophysical Research Letters, 37(6), L06,305, doi:10.1029/2009GL041869.

Ismail-Zadeh, A., G. Schubert, I. Tsepelev, and A. Korotkii (2004), Inverse problem of thermal convection: numerical approach and application to mantle plume restoration, Physics of the Earth and Planetary Interiors, 145(1-4), 99-114, doi:10.1016/j.pepi.2004.03. 006.

Kalnay, E. (2003), Atmospheric modeling, data assimilation and predictability, Cambridge University Press.

Kaula, W. M. (1980), Material properties for mantle convection consistent with observed surface fields, Journal of Geophysical Research, 85(B12), 7031, doi: 10.1029/JB085iB12p07031.

Liu, L., S. Spasojevic, and M. Gurnis (2008), Reconstructing Farallon plate subduction beneath North America back to the Late Cretaceous., Science, 322(5903), 934-8, doi:10.1126/science.1162921.

Lorenz, E. N. (1963), Deterministic Nonperiodic Flow, Journal of the Atmospheric Sciences, 20(2), 130-141, doi:10.1175/1520-0469(1963)020\$<\$0130: $\mathrm{DNF} \$ \$ 2.0 . \mathrm{CO} ; 2$.

Lorenz, E. N. (1965), A study of the predictability of a 28-variable atmospheric model, Tellus, 17(3), 321333, doi:10.1111/j.2153-3490.1965.tb01424.x.

Mattern, E., J. Matas, Y. Ricard, and J. Bass (2005), Lower mantle composition and temperature from mineral physics and thermodynamic modelling, Geophysical Journal International, 160(3), 973-990, doi:10. 1111/j.1365-246X.2004.02549.x.

McNamara, A. K., and S. Zhong (2005), Thermochemical structures beneath Africa and the Pacific Ocean., Nature, 437(7062), 1136-9, doi:10.1038/ nature04066.

Mitrovica, J., and A. Forte (2004), A new inference of mantle viscosity based upon joint inversion of convection and glacial isostatic adjustment data, Earth and Planetary Science Letters, 225(1-2), 177-189, doi: 10.1016/j.epsl.2004.06.005.

Moucha, R., and A. M. Forte (2011), Changes in African topography driven by mantle convection, Nature Geoscience, 4(10), 707-712, doi:10.1038/ngeo1235.

Ricard, Y., and B. Wuming (1991), Inferring the viscosity and the 3-D density structure of the mantle from geoid, topography and plate velocities, Geophysical Journal International, 105(3), 561-571, doi: 10.1111/j.1365-246X.1991.tb00796.x.

Ricard, Y., M. Richards, C. Lithgow-Bertelloni, and Y. Le Stunff (1993), A geodynamic model of mantle density heterogeneity, Journal of Geophysical Research, 98(B12), 21,895, doi:10.1029/93JB02216.

Rolf, T., and P. J. Tackley (2011), Focussing of stress by continents in 3D spherical mantle convection with selfconsistent plate tectonics, Geophysical Research Letters, 38(18), L18,301, doi:10.1029/2011GL048677.

Sanchez, S., A. Fournier, and J. Aubert (2014), The predictability of advection-dominated flux-transport solar dynamo models, The Astrophysical Journal, 781:8(1), 1-15, doi:10.1088/0004-637X/781/1/8.

Seton, M., R. Müller, S. Zahirovic, C. Gaina, T. Torsvik, G. Shephard, A. Talsma, M. Gurnis, M. Turner, S. Maus, and M. Chandler (2012), Global continental and ocean basin reconstructions since 200Ma, EarthScience Reviews, 113(3-4), 212-270, doi:10.1016/j. earscirev.2012.03.002.

Steinberger, B., and R. J. O'Connell (1997), Changes of the earth's rotation axis owing to advection density heterogeneities, Nature, 387, 169-173.

Stewart, C. A., and D. L. Turcotte (1989), The route to chaos in thermal convection at infinite Prandtl number: 1 . Some trajectories and bifurcations, Journal of Geophysical Research, 94(B10), 13,707, doi: 10.1029/JB094iB10p13707.

Stixrude, L., and C. Lithgow-Bertelloni (2007), Influence of phase transformations on lateral heterogeneity and dynamics in Earth's mantle, Earth and Planetary Science Letters, 263(1-2), 45-55, doi:10.1016/j.epsl. 2007.08.027.

Tackley, P. J. (1996), On the ability of phase transitions and viscosity layering to induce long wavelength Heterogeneity in the mantle, Geophysical Research Letters, 23(15), 1985-1988, doi:10.1029/96GL01980.

Tackley, P. J. (2008), Modelling compressible mantle convection with large viscosity contrasts in a threedimensional spherical shell using the yin-yang grid, 
Physics of the Earth and Planetary Interiors, 171(1-4), 7-18, doi:10.1016/j.pepi.2008.08.005.

Travis, B., and P. Olson (1994), Convection with internal heat sources and thermal turbulence in the Earth's mantle, Geophysical Journal International, 118(1), 119, doi:10.1111/j.1365-246X.1994.tb04671.x.

Van Heck, H. J., and P. J. Tackley (2008), Planforms of self-consistently generated plates in $3 \mathrm{D}$ spherical geometry, Geophysical Research Letters, 35(19), L19,312, doi:10.1029/2008GL035190.

Weeraratne, D., and M. Manga (1998), Transitions in the style of mantle convection at high Rayleigh numbers, Earth and Planetary Science Letters, 160(3-4), 563568, doi:10.1016/S0012-821X(98)00111-3.

Yoshida, M., and M. Santosh (2011), Supercontinents, mantle dynamics and plate tectonics: A perspective based on conceptual vs. numerical models, Earth-Science Reviews, 105(1-2), 1-24, doi:10.1016/ j.earscirev.2010.12.002.

Zhang, N., and S. Zhong (2011), Heat fluxes at the Earth's surface and core-mantle boundary since Pangea formation and their implications for the geomagnetic superchrons, Earth and Planetary Science Letters, 306(3-4), 205-216, doi:10.1016/j.epsl.2011. 04.001 .

Zhang, N., S. Zhong, W. Leng, and Z.-X. Li (2010), A model for the evolution of the Earth's mantle structure since the Early Paleozoic, Journal of Geophysical Research, 115(B6), B06,401, doi:10.1029/ 2009JB006896.

Zhong, S., M. T. Zuber, L. Moresi, and M. Gurnis (2000), Role of temperature-dependent viscosity and surface plates in spherical shell models of mantle convection, Journal of Geophysical Research, 105(B5), 11,06311,082, doi:10.1029/2000JB900003.

Ziehmann, C., L. A. Smith, and J. Kurths (2000), Localized Lyapunov exponents and the prediction of predictability, Physics Letters A, 271(4), 237-251, doi: 10.1016/S0375-9601(00)00336-4. 
(a) mantle (ISO)

(b) Layered
viscosity
(LV)

(c) Plate-like
behavior (PL)

(d) Plate-like
behavior
+
Layered
viscosity
(PLLV)

(e)

Plate-like
behavior
+
continents
(PLC)
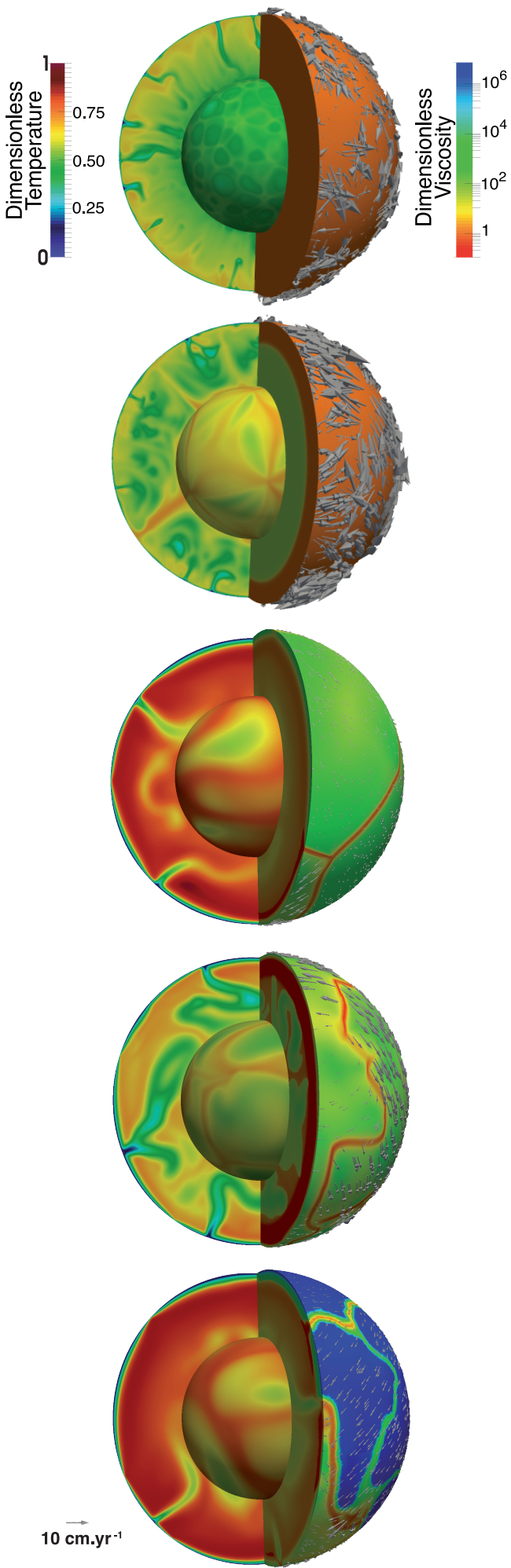
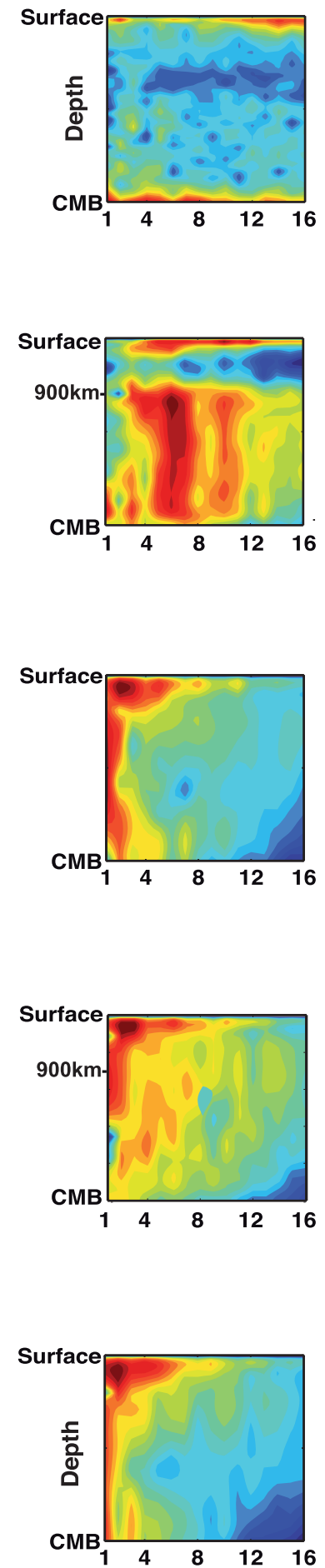

Spherical Harmonic degree

Figure 1: Snapshots of the interior temperature field (left side), viscosity and surface velocity (right side), and spherical harmonic maps of the initial state of convection calculations for five different rheologies. White arrows represent surface velocities. Each panel of spherical harmonic map is normalized to the maximum amplitude. The values increase exponentially from dark blue to dark red and there are 20 contour intervals. (a) Isoviscous mantle, $\mathrm{Ra}=10^{7}$. (b) Mantle with a viscosity increase by a factor of 40 between 800 and $1000 \mathrm{~km}$ depth, Ra=10 ${ }^{7}$. (c) Mantle with temperature-dependent viscosity and pseudo-plastic yielding, $R a=10^{6}$. (d) Same as (c) with a viscosity increase by a factor of 30 between 800 and $1000 \mathrm{~km}$ depth, $\mathrm{Ra}=10^{6}$. (e) Same as (c) with continents. All calculations are internally heated only. 


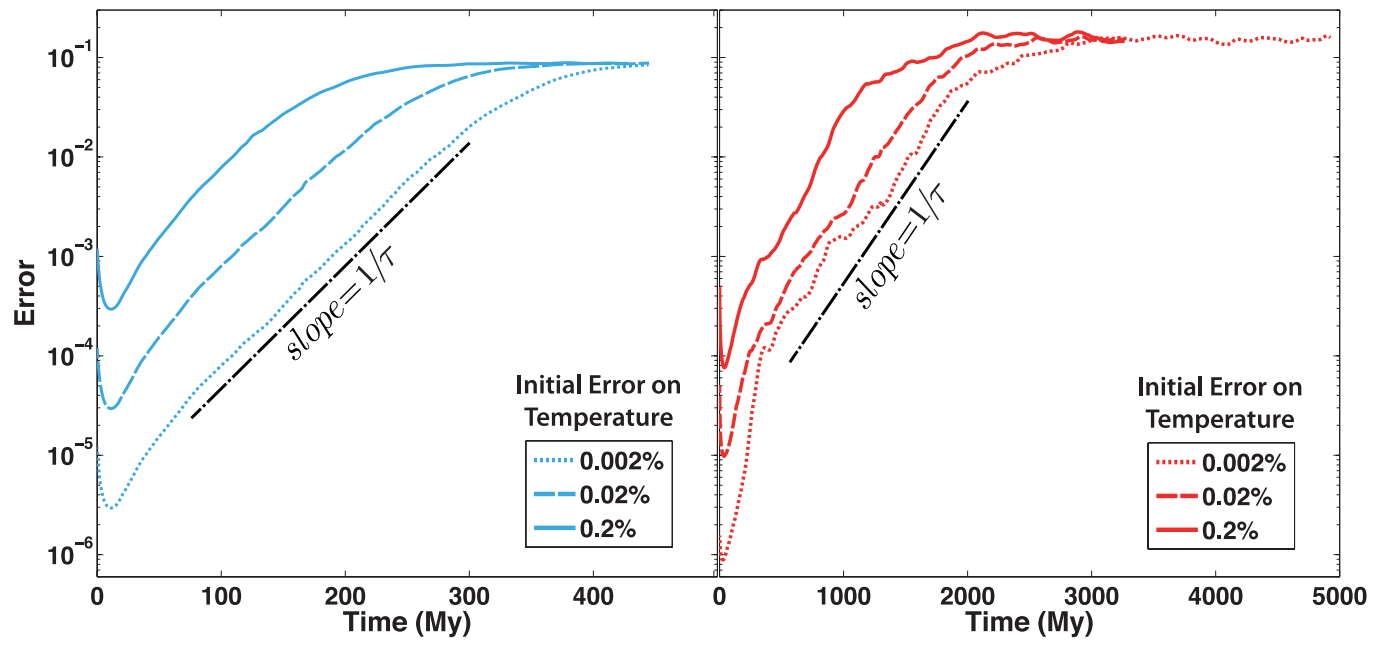

Figure 2: Error growth for three twin experiments started from the same reference temperature field, for two different rheologies: isoviscous ISO4,5,6 (left) and pseudo-plastic yielding PL6,7,8 (right). $E(0)$, the initial error on the temperature of the twin experiment was alternatively set to $0.2 \%$ (bold line), $0.02 \%$ (dashed line) and $0.002 \%$ (dotted line). The error on the dimensionless temperature is plotted versus time. Note that the time scales on the $x$-axis is different. The resulting Lyapunov times $\tau$ are obtained fitting the slope of the phase of exponential growth. 


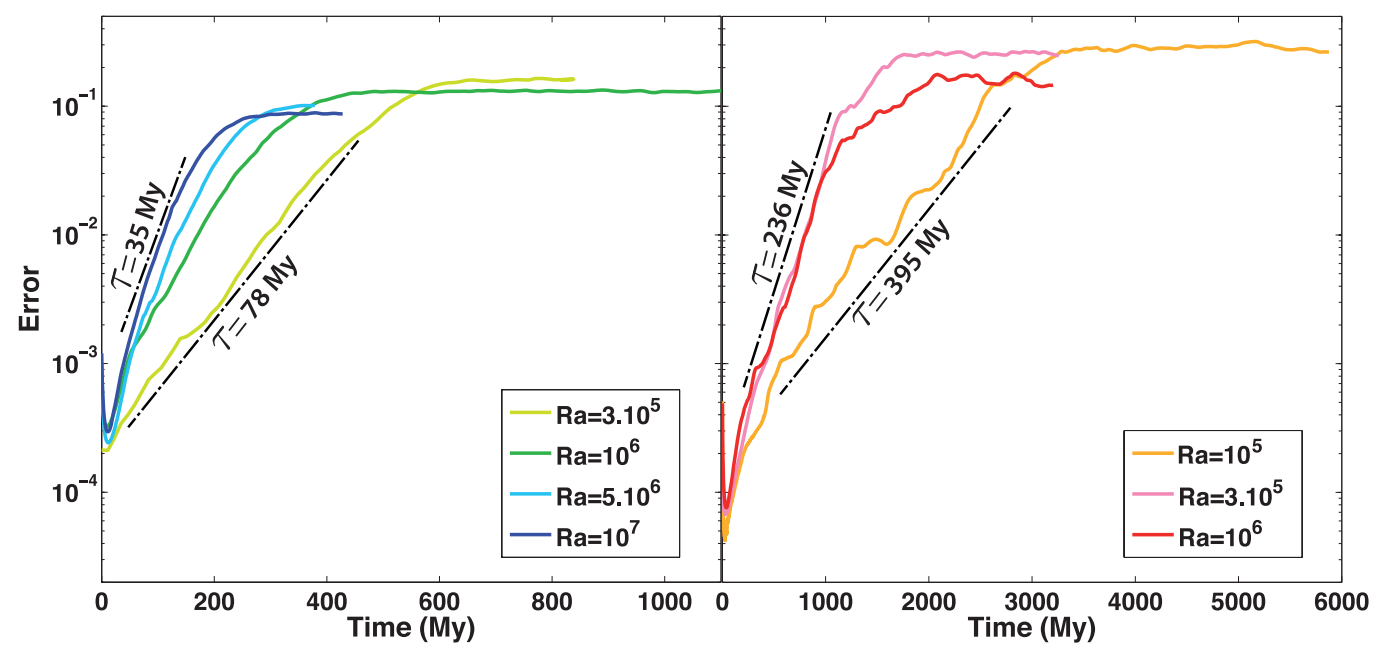

Figure 3: Error growth for twin experiments varying only in the Rayleigh number. Each panel displays the results for a given viscosity law: isoviscous (ISO1,2,3,6) with Ra ranging from $3 \times 10^{5}$ to $10^{7}$ (left) and pseudo-plastic yielding $(\mathrm{PL} 2,5,8)$ with Ra ranging from $10^{5}$ to $10^{6}$ (right). $E(0)=0.2 \%$ for all experiments. 


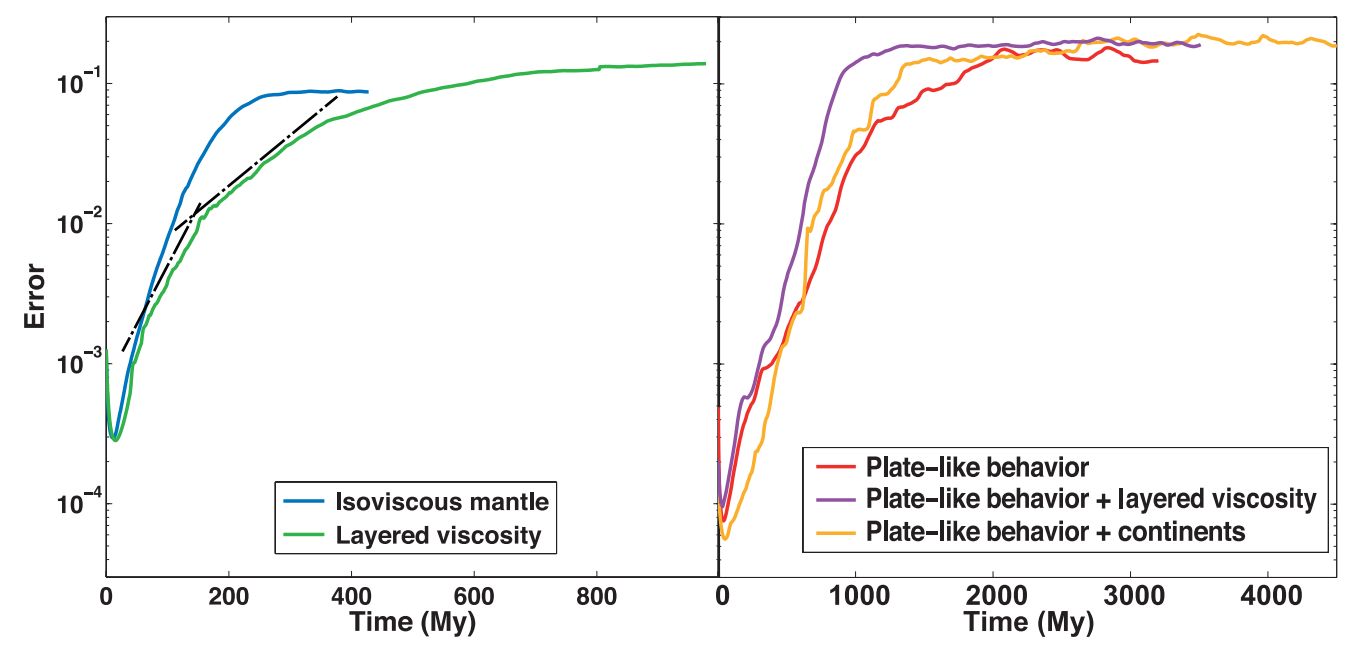

Figure 4: Error growth for different rheologies: isoviscous ISO6 (blue), layered viscosity with an increase by a factor of 40 between 800 and $1000 \mathrm{~km}$ depth LV2 (green), pseudo-plastic yielding only PL8 (red), pseudo-plastic yielding combined to a viscosity increase by a factor of 30 between 800 and $1000 \mathrm{~km}$ depth PLLV3 (purple), and pseudo-plastic yielding with continents PLC3 (orange). $E(0)$ is $0.2 \%$ for all experiments. The Rayleigh number is chosen so as to ensure fully chaotic behavior: $\mathrm{Ra}=10^{7}$ for ISO and LV, Ra $=10^{6}$ for PL, PLLV and PLC. 
Table 1: Convection parameters and Lyapunov times of the twin experiments computed for this study.

\begin{tabular}{|l|l|r|r|r|c|}
\hline Name & Rheological Model & \multicolumn{1}{c|}{$\mathrm{Ra}$} & \multicolumn{1}{c|}{$\mathrm{H}(0)(\%)$} & $\tau(\mathrm{My})$ \\
\hline (ISO1) & Isoviscous & $3 \times 10^{5}$ & 13.42 & 0.2 & 77.8 \\
(ISO2) & Isoviscous & $10^{6}$ & 20.05 & 0.2 & 55.3 \\
(ISO3) & Isoviscous & $5 \times 10^{6}$ & 34.28 & 0.2 & 39.5 \\
(ISO4) & Isoviscous & $10^{7}$ & 44.17 & 0.002 & 34.8 \\
(ISO5) & Isoviscous & $10^{7}$ & 44.17 & 0.02 & 35.6 \\
(ISO6) & Isoviscous & $10^{7}$ & 44.17 & 0.2 & 35.0 \\
\hline (LV1) & Layered Viscosity & $10^{7}$ & 44.17 & 0.2 & $59.8 / 116$ \\
(LV2) & Layered Viscosity & $10^{7}$ & 44.17 & 0.2 & $54.8 / 121$ \\
\hline (PL1) & Plate-Like Behavior & $10^{5}$ & 9.52 & 0.02 & 466 \\
(PL2) & Plate-Like Behavior & $10^{5}$ & 9.52 & 0.2 & 395 \\
(PL3) & Plate-Like Behavior & $3 \times 10^{5}$ & 13.42 & 0.002 & 200 \\
(PL4) & Plate-Like Behavior & $3 \times 10^{5}$ & 13.42 & 0.02 & 190 \\
(PL5) & Plate-Like Behavior & $3 \times 10^{5}$ & 13.42 & 0.2 & 239 \\
(PL6) & Plate-Like Behavior & $10^{6}$ & 20.05 & 0.002 & 265 \\
(PL7) & Plate-Like Behavior & $10^{6}$ & 20.05 & 0.02 & 247 \\
(PL8) & Plate-Like Behavior & $10^{6}$ & 20.05 & 0.2 & 196 \\
\hline (PLLV1) & Plate-Like Behavior \& Layered Viscosity & $10^{6}$ & 20.05 & 0.002 & 124 \\
(PLLV2) & Plate-Like Behavior \& Layered Viscosity & $10^{6}$ & 20.05 & 0.02 & 113 \\
(PLLV3) & Plate-Like Behavior \& Layered Viscosity & $10^{6}$ & 20.05 & 0.2 & 119 \\
\hline (PLC1) & Plate-Like Behavior \& Continents & $10^{6}$ & 20.05 & 0.002 & 131 \\
(PLC2) & Plate-Like Behavior \& Continents & $10^{6}$ & 20.05 & 0.02 & 149 \\
(PLC3) & Plate-Like Behavior \& Continents & $10^{6}$ & 20.05 & 0.2 & 128 \\
\hline
\end{tabular}

